\title{
A Importância da Responsabilidade Social Corporativa para a Potenciação do Capital Social em Pequenas e Médias Empresas
}

\author{
Maria João Nicolau Santos ${ }^{1}$ \\ Rogério Rodrigues da Silva ${ }^{2}$
}

\section{Resumo}

As transformações sociais, econômicas e tecnológicas têm transformado o papel das empresas perante a sociedade e sob esse prisma a questão da Responsabilidade Social Corporativa assume um papel importante. Todavia para as pequenas $e$ médias empresas existem limitações que impedem uma integração maior desse tipo de prática na sua gestão. Para superar isso, tem-se buscado uma integração maior dessas empresas aos stakeholders, a custo baixo, a fim de maximizarem as possíveis vantagens, o que está imbricado no conceito de capital social. Assim, o objetivo deste artigo é apresentar a teoria do capital social e relacioná-la a questões de Responsabilidade Social Corporativa, buscando compreender as intersecções, aplicabilidade e ganhos desses conceitos no contexto das pequenas e médias empresas. Indicam-se ainda algumas variáveis que podem servir como base para mensuração da Responsabilidade Social Corporativa e do capital social em pesquisas futuras.

Palavras-chave: Responsabilidade social. Capital social. Pequenas e médias empresas.

\section{Introdução}

As transformações sociais, econômicas e tecnológicas, sobretudo das duas últimas décadas, criaram um cenário que favorece o questionamento

\footnotetext{
${ }^{1}$ Doutora em Sociologia Económica e das Organizações. Professora Auxiliar no Instituto Superior de Economia e Gestão (ISEG) da Universidade Técnica de Lisboa. Investigadora do Centro de Investigação em Sociologia Económica e das Organizações (SOCIUS). Membro do Conselho Cientifico do Programa de Doutoramento. Coordenadora do Mestrado em Gestão de Recursos Humanos no Instituto Superior de Economia e Gestão (ISEG- UTL). Endereço: Instituto Superior de Economia e Gestão (ISEG) da Universidade Técnica de Lisboa, Rua Miguel Lupi, n 20 , CEP: 1249-078, Lisboa-Portugal.E-mail: mjsantos@iseg.ut.pt.

${ }^{2}$ Psicólogo. Especialista em Gestão de Pessoas. Mestre em Psicologia Social e do Trabalho pela Universidade de Brasilia. Doutorando em Sociologia Económica e das Organizações pelo Instituto Superior de Economia e Gestão da Universidade Técnica de Lisboa. Endereço: Instituto Superior de Economia e Gestão (ISEG) da Universidade Técnica de Lisboa, Rua Miguel Lupi, n 20, CEP: 1249-078, Lisboa - Portugal. E-mail: rogeriopsi@hotmail.com.

Artigo recebido em: 14/07/2008. Aceito em: 30/11/2009. Membro do Corpo Editorial Científico responsável pelo processo editorial: Gilberto de Oliveira Moritz.
} 
da relação entre Estado, empresas e sociedade. Nesse novo cenário, várias implicações podem ser elencadas como a internacionalização do mercado, o aumento do tamanho e da influência das empresas, a intervenção estatal para desfazer o estado de bem-estar social, a luta cada vez maior por expertise, entre outras (CASTELLS, 1999).

Diante disso, os critérios para avaliação de eficiência e sucesso das organizações passam a considerar não apenas aspectos de natureza econômica, mas também de natureza social, cultural e ambiental. Além disso, pressionadas pela própria sociedade, as empresas passam também a assumir uma responsabilidade maior pelo bem-estar da sociedade e fornecem garantias que antes estavam atreladas apenas ao Estado, tais garantias são chamadas hoje de Responsabilidade Social.

Embora seja uma questão observada em um grande número de empresas, poucos estudos têm sido feitos sobre esse tipo de prática em pequenas e médias empresas - PMEs. No Brasil, o critério mais usual para definição do porte das empresas, segundo o SEBRAE (2005), está relacionado ao número de seus empregados: a) Pequena empresa: na indústria, de 20 a 99 pessoas ocupadas e no comércio e serviços, de 10 a 49 pessoas ocupadas; e b) Média empresa: na indústria, de 100 a 499 pessoas ocupadas e no comércio e serviços, de 50 a 99 pessoas ocupadas. As PMEs encerram uma especificidade própria que as distingue das empresas de grande dimensão. A afirmação da responsabilidade social decorre de uma complexa rede de forças, de interesses e de convicções, dividida fundamentalmente entre, por um lado, a afirmação de um "espírito empresarial responsável", assumido geralmente pelo empresário e, por outro lado, a existência de um não menos influente conjunto de pressões externas. A participação no mercado internacional, frequentemente pela via da subcontratação, obriga ao cumprimento de padrões previamente definidos pelas multinacionais como condição para operarem. A pressão das instituições reguladoras e dos competidores, bem como os incentivos financeiros dos governos são igualmente fatores de estímulo e/ou pressão para uma maior atuação a esse nível. Verifica-se também que as dinâmicas e as práticas assumidas são menos explicitas, pois têm um caráter informal, não estruturado, assumindo geralmente um aspecto ocasional, não estando integradas na estratégia da empresa (SANTOS et al., 2006).

Segundo Habisch (2004), esse tipo de empresa tem sido o sustentáculo da responsabilidade social corporativa nas comunidades onde atuam, embora enfrentem uma série de barreiras para realizarem algum tipo de ação 
dessa natureza. Para Southwell (2004) e Santos et al. (2006), essas barreiras apontam para a falta de tempo e recursos financeiros e, principalmente, a falta de conhecimento dos instrumentos de apoio, monitoramento e validação. A inexistência de ferramentas eficazes de avaliação da performance impede que se ultrapasse o obstáculo associado à dificuldade de testar se podem advir perdas de competitividade perante outras organizações que não assumem idêntica orientação.

Para superarem essas limitações, faz-se necessária uma reorganização da empresa para estar cada vez mais atenta à demanda de seus stakeholders e do mercado como um todo. Castells (1999) salienta a esse propósito que a superação das dificuldades encontradas pelas PMEs passa pela sua integração em redes de cooperação. Independente do tipo de associação (redes interempresariais ou relacionamentos de parceria, por exemplo), o importante é referir que agir isoladamente implica grandes dificuldades. Assim, importa ter uma melhor compreensão sobre o papel desses relacionamentos, sobretudo, da confiança e das vantagens estabelecidas, o que pode ser atribuído ao capital social (ARAÚJO, 2003). Para Werner e Spence (2004), esse tipo de capital pode ser visto como um caminho de prosperidade econômica e de desenvolvimento sustentável para as PMEs. Segundo Ayuso et al. (2006), a capacidade das empresas integrarem, na ótica do desenvolvimento sustentável, os insights dos stakeholders no processo de inovação organizacional, constitui um fator de relevância extrema, gerador de forte vantagem competitiva. No entanto, as abordagens correntes não consideram o potencial das redes de capital social no entendimento das mudanças globais e, sobretudo, no processo de inovação empresarial.

Diante disso, o que se busca neste artigo é mostrar como a questão da responsabilidade social pode potencializar o capital social das empresas por meio de uma articulação mais estreita com os stakeholders externos e das dinâmicas positivas que isso gera ao nível dos stakeholders internos, sobretudo, entre trabalhadores/colaboradores. Sob esse pressuposto, e com base nas investigações efetuadas, algumas questões de partida formam o presente artigo. Será que as empresas se beneficiam por realizarem ações socialmente responsáveis? Até que ponto níveis maiores de confiança, compartilhamento de normas, de valores, de crenças podem significar algum tipo de vantagem para as empresas? Complementarmente, será que as ações socialmente responsáveis podem de alguma forma afetar os níveis de cooperação e confiança estabelecidos com os stakeholders ou será que é o contrário? São essas questões que o presente artigo propõe discutir. 


\section{Responsabilidade Social em Pequenas e Médias Empresas}

A Responsabilidade Social Corporativa - RSC pode ser definida como sendo os modos formais e informais em que os negócios dão sua contribuição para melhorar a governança $e$ as condições sociais, éticas, de trabalho $e$ também ambientais dos países em que operam, enquanto estão sensíveis para seus contextos religiosos, históricos e culturais (VISSER, 2008). Nessa mesma direção, Perrini (2006) advoga que as questões econômicas, sociais e ambientais devem estar igualmente integradas às estratégias de negócios, às ferramentas de gerenciamento e a todas as atividades das empresas, o que se torna um grande desafio para as empresas de pequeno ou médio porte.

Trata-se fundamentalmente de um modelo diferenciado de gestão da atividade de negócio. Pressupõe, dessa forma, um gerenciamento contínuo que toma em consideração e incorpora ao planejamento estratégico da empresa as preocupações com a sustentabilidade da sociedade, a preservação dos recursos naturais e culturais. Como refere o antigo Secretário Geral das Nações Unidas, Kofi Annan, não se pede às empresas para fazer algo diferente da sua atividade normal, mas para fazerem a sua atividade normal de forma diferente (SANTOS et al., 2006).

Essa mudança na forma de gerenciar o negócio pressupõe a assunção de um compromisso no qual a implementação de processos produtivos $e$ comerciais passa a estar baseada no estabelecimento de relações éticas, transparentes e solidárias da empresa com todos os seus stakeholders. Contudo, essas relações não se mostram fáceis de serem alcançadas para as PMEs, já que estão envolvidos expertise, planejamento de longo prazo e ainda um grande leque de atores cujas demandas podem tornar inviável qualquer iniciativa que transpasse o aspecto legal. Além disso, a falta de acesso ao crédito, a incapacidade de negociar as práticas de RSC com clientes e fornecedores, a falta de motivação de empregados e, principalmente, a ausência, quase que completa, de ferramentas de mensuração e quantificação dos benefícios solapam muitas das iniciativas das $\mathrm{PMEs}$ em relação à responsabilidade social corporativa (TEIXEIRA, 1996; SPENCE; SCHMIDPETER, 2003; PERRINI, 2006; SANTOS et al., 2006; JENKINS, 2006).

Todavia, estudos apontam algumas peculiaridades que podem se configurar como facilidades para a integração de RSC à gestão dessas empresas, são elas: a estratégia informal de relacionamento com os stakeholders, pau- 
tada principalmente no diálogo mais permanente; uma burocracia pequena; um grande potencial de assimilação, adaptação e geração de novas tecnologias (CASTELLS, 1999; BALESTRIN; VARGAS, 2004; GRAAFLAND; VEN; STOFFELE, 2003; SPENCE; SCHMIDPETER, 2003; PERRINI, 2006; JENKINS, 2006).

No contexto brasileiro, a motivação maior das ações de RSC das PMEs volta-se para a filantropia e o assistencialismo, o que não reflete uma preocupação com a minimização ou a transformação dos problemas sociais (PELIANO, 2002). No Brasil, as ações socialmente responsáveis das PMEs são orientadas para a melhoria da imagem institucional, para a manutenção de uma política de boa vizinhança e para o aumento da produtividade (TEIXEIRA, 1996; PELIANO, 2002). Uma das razões que podem explicar essa ênfase é que no Brasil a falta de integração interempresarial, a burocratização e a ausência de redes (sociais ou empresariais) efetivas impedem uma maior cooperação entre as empresas (FUKUYAMA, 1995; MELO NETO; FROES, 2001). Caso fossem superadas tais razões, as atividades sociais das empresas teriam um alcance muito maior e, principalmente, os níveis de confiança entre empresas e seus stakeholders seriam aumentados (PELIANO, 2002).

Já no contexto europeu, as pesquisas apontam que, para além do envolvimento com a comunidade, predomina um forte envolvimento das PMEs com os empregados (desenvolvimento de pessoal, responsabilidade pelo bem-estar e saúde dos funcionários) e as atividades de caráter ambiental (redução de impacto ambiental, reciclagem e redução de lixo) (GRAAFLAND et al., 2003; SOUTHWELL, 2004; SANTOS et al., 2006). Estudos em países europeus apontam ainda que o aumento de RSC entre as PMEs europeias deve-se, sobretudo, à busca de melhoria da reputação, de maiores níveis de satisfação dos colaboradores, mas também por conta da oportunidade de fazer novas parcerias, sobretudo, com empresas de grande porte. Isso se deve especialmente ao fato de que nos contratos com empresas grandes há exigências pelo cumprimento de determinados padrões sociais e ambientais, o que impulsiona a RSC entre as PMEs (SOUTHWELL, 2004; FULLER; TIAN, 2006; SANTOS et al., 2006). Além disso, essas práticas estão ainda associadas à redução de custos, à melhoria do serviço prestado ao cliente, aos incrementos na qualidade do produto ofertado e à tentativa de aumento na eficácia da gestão ambiental (UNIDO, 2002; SANTOS et al., 2006).

Embora pareçam bastante distintas, as motivações europeias e brasileiras encontram uma intersecção no que tange às motivações. De modo geral, 
as pesquisas indicam que para além dessas questões decorrentes das pressões do mercado, as motivações giram, tanto no contexto brasileiro quanto no europeu, em torno de questões de ordem moral, ética e até religiosa, tal como "fazer a coisa certa", "sentir-se bem" ou "devolver algo para a comunidade" (TEIXEIRA, 1996; JENKINS, 2006; WORTHINGTON; RAM; JONES, 2006).

No que se refere às vantagens de uma gestão socialmente responsável, em estudo com empresas inglesas, Jenkins (2006) aponta que não houve indícios de uma relação significativa entre benefícios sociais e benefícios econômicos, contudo, a autora ratifica evidências no que se refere à motivação do empregado e à melhoria da imagem da empresa. Estudos portugueses reiteram, além disso, que a RSC em PMEs está diretamente associada ao aumento da satisfação dos trabalhadores, à fidelização de clientes, mas principalmente à redução de custos (REGO, 2003; SANTOS et al., 2006). Ademais, estudos apontam ainda que a RSC para as PMEs pode servir como uma estratégia que potencializa o acesso a novos mercados consumidores e ainda reforça as relações comerciais já estabelecidas com outras empresas, clientes e fornecedores (UNIDO, 2002; HABISCH, 2004).

Diante do exposto, pode-se inferir que o desenvolvimento de uma gestão socialmente responsável em PMEs é desafiante. Além de representarem mais de 95\% das empresas existentes no Brasil (PELIANO, 2002), algumas de suas características beiram a dicotomia entre limitação e potencialidade. As ferramentas para o desenvolvimento dessa gestão não passam, portanto, simplesmente por uma adaptação do conhecimento gerado em grandes empresas, mas pela caracterização da diversidade das pequenas e médias e pelo desenvolvimento de instrumentos de ação especialmente orientados para essa realidade. Uma das saídas apontadas volta-se principalmente para a troca constante com outros atores sociais e econômicos, como é o caso da inserção dessas empresas em redes de cooperação (SPENCE; SCHMIDPETER, 2003; VERSCHOORE, 2004). Nessa perspectiva, o conceito de redes em responsabilidade social (ZADEK, 2001) ganha atualmente maior visibilidade, surgindo como um instrumento de atuação para alavancar a responsabilidade corporativa a níveis meso e macro, ultrapassando a visão restritiva de impactos limitados e permitindo, por essa via, atingir até mesmo benefícios competitivos de caráter nacional.

A criação dessas redes de parceria social ganha, no atual contexto de crise econômica, uma importância maior. A complexidade das situações e a escassez de recursos financeiros não possibilitam que isoladamente uma or- 
ganização, sobretudo uma pequena empresa, possa intervir de forma adequada na resolução de problemas sociais, econômicos, ou mesmo, ambientais. Acresce que constitui também um importante fator de inovação, pois o diálogo e a integração do conhecimento dos stakeholders, em uma ótica de desenvolvimento sustentável, permitem gerar mudanças na forma de operar, bem como nos produtos e serviços providenciados. Destarte, as parcerias interempresariais surgem como instrumento de ação fundamental que permitem agregar sinergias e potencializar ações que ultrapassam o âmbito de cada organização tomada individualmente. Essa saída parte da premissa de que RSC não se opera em um vácuo desconectado do mundo, antes, inteiramente ligada e conectada à demanda de seus stakeholders.

\section{Capital Social e Redes de Cooperação}

No que tange às pequenas e médias empresas, as trocas estabelecidas com outros atores sociais e econômicos têm se tornado cada vez mais necessárias para sua sobrevivência no mercado e estão imbricadas no desenvolvimento do que se chama capital social. Esse conceito refere-se ao acesso a recursos disponíveis como uma consequência da participação em redes de suporte e obrigações mútuas que tornam possível as ações coletivas entre seus participantes (ARAÚJO, 2003).

Para Pierre Bourdieu (1986), capital social é o conjunto de relações e redes de ajuda mútua que podem ser mobilizadas efetivamente para beneficiar o indivíduo ou sua classe social. Assim, a ideia de capital social remete aos recursos resultantes da participação em redes de relações de conhecimento e reconhecimento institucionalizadas ou não, a fim de satisfazer às necessidades dos grupos.

Esse conceito de capital social tem sido objeto de estudo de sociólogos, economistas, administradores e até de psicólogos. James Coleman (1988), por exemplo, referiu-se ao papel do capital social no desenvolvimento do capital humano. Robert Putnam (2000), com seus estudos sobre a terceira Itália, aponta para a importância do engajamento social no desenvolvimento regional ou nacional. Mark Granovetter (1973) afirma que as relações flexíveis podem ajudar muito os indivíduos e estão ligadas ao tempo, à intensidade emocional e à intimidade das trocas entre os atores. Já Ronald Burt (2000) advoga que as pessoas podem se beneficiar como intermediárias em termos de informação e controle das vantagens obtidas nas redes pessoais. 
A aplicação desse conceito de capital social à realidade das PMEs baseia-se em dois pontos. A primeira se refere ao fato de que essas empresas já estão fortemente imbricadas em redes locais, em comunidades e em outras relações pessoais, permitindo o desenvolvimento de relacionamentos muito próximos (fornecedores, clientes e empregados). Outro ponto é que as relações individuais e organizacionais desenvolvidas pelas PMEs podem ser mutuamente reforçadoras (SPENCE; SCHMIDPETER, 2003; WORTHINGTON; RAM; JONES, 2006), ou seja, quanto mais uma empresa se envolve com outros atores, mais esses atores se envolvem com essa empresa.

O caráter eminentemente social desse conceito traz alguns complicadores para sua mensuração e experimentação, visto que está baseado em relações humanas e, por isso, não é facilmente transferível. Diante desse impasse, é necessário compreender os principais constituintes desse conceito, sobretudo para identificar os diferentes tipos de envolvimento, diálogo com stakeholders ou integração do conhecimento dos stakeholders e ainda mensurar esse envolvimento de pessoas ou de empresas em relações sociais ou redes sociais.

Para Adler e Kwon (2000), capital social é constituído necessariamente por: a) redes sociais; b) normas compartilhadas e; c) crenças compartilhadas entre pessoas, grupos ou organizações. Para esses autores, as redes (de pessoas, grupos ou organizações) maximizam a possibilidade de acesso ao suporte entre os atores e ainda aos recursos mobilizados pela rede. As normas compartilhadas trazem significado ao contexto do relacionamento, o que facilita a ação coletiva ou individual, e as crenças permitem aos participantes comunicarem suas ideias e fazer com que as experiências comuns tenham sentido, acarretando visões e expectativas comuns.

Merece destaque também, sob essa orientação, o papel que a confiança tem nas redes estabelecidas. Confiança pode ser definida como uma expectativa de que o parceiro de negócio se comportará de maneira aceitável (SAKO, 1998). É essa confiança que permite o cumprimento das obrigações e das normas estabelecidas nos relacionamentos interorganizacionais (COLEMAN, 1988; WERNER; SPENCE, 2004). Adler e Kwon (2000) advogam que quanto mais as pessoas participam de comunidades, de grupos ou de redes, mais elas aprendem a confiar e quanto maior é essa confiança, mais abertas estão para participarem.

Aplicados às redes de cooperação firmadas entre as PMEs, os estudos apontam para três benefícios no desenvolvimento do capital social (BURT, 2000; ADLER; KWON, 2000; FUKUYAMA, 2001; VERSCHOORE, 2004): 
a) Facilidade de acesso a maiores fontes de informações com custo menor. No caso das pequenas e médias empresas, essas fontes podem significar oportunidades maiores de negócios, redução de custos com fornecedores, aquisição de novas habilidades e conhecimentos, formação de parcerias com outras empresas, entre outros.

b) Poder e influência. Os detentores de informações privilegiados têm poder de controle. Adler e Kwon (2000) apontam que ter poder e influência possibilita que as pessoas liderem outras por meio de objetivos comuns, o que facilita a ação coletiva. Além disso, Verschoore (2004) aponta que empresas em redes, por exemplo, têm maior confiança no mercado e, por isso, poder de acesso ao crédito.

c) Unidade. Diante do contato constante entre os atores, das normas e crenças compartilhadas, há uma maior facilidade de resolução de problemas, de queixas, de engajamento cívico. Além disso, segundo Adler e Kwon (2000), normas e crenças fortes, associadas a alto grau de unidade, encorajam submissão às regras e costumes e ainda reduzem a necessidade de controles formais. Aliás, o excesso desses controles formais diminui consideravelmente a possibilidade de sucesso no estabelecimento de boas relações de confiança ou pessoais (RING; VAN DE VEN, 1994; SAKO, 1998).

Para se ter uma ideia do que isso significa na prática, Verschoore (2004) relata que a Secretaria de Desenvolvimento e Assuntos Internacionais SEDAI, do Rio Grande do Sul, desenvolve um projeto para redes de cooperação desde o ano 2000. Esse projeto tem conseguido resultados expressivos para o desenvolvimento e o fortalecimento de pequenas e médias empresas naquele estado, contando hoje com mais de 160 redes de cooperação, e com mais de 4.000 empresas associadas. Segundo o autor, o sucesso dessas redes se deve principalmente às vantagens econômicas relacionadas à diminuição de custo que gira em torno dos $36 \%$, depois da associação à rede. Outra vantagem apontada pelo autor é o aumento do faturamento que, no exemplo da Associação dos Fabricantes de Estofados e Móveis Complementares, uma das redes associadas, pulou de $R \$ 37$ milhões para $R \$ 76$ milhões depois de cinco anos de associação em rede.

Esses resultados obtidos no Rio Grande do Sul não passam apenas pelo estabelecimento de redes em si, mas principalmente pelo tipo de relaciona- 
mento aí desenvolvido. Contratos de associação com regras, obrigações e direitos bastante claros facilitam essa integração cada vez maior para os participantes das redes. Contudo, há de se dizer que, tal como ressaltam Ring e Van de Ven (1994), é necessário um balanceamento entre a formalização contratual e os contatos pessoais, já que se a relação na rede for muito formal haverá engessamento da rede. Bem como se a rede se pautar exclusivamente em relações informais entre os participantes, haverá o declínio rápido dessa rede.

Montana e Nenide (2008), por exemplo, mostraram, em estudo na região de San Joaquin Valley - Califórnia, Estados Unidos, que as redes integraram mais empregos à região que a média regional e nacional. Além disso, as empresas que compõem os dez clusters da região cresceram em média mais de $10 \%$ no período de cinco anos, enquanto a média regional foi de $8 \%$. Além disso, o cluster ligado ao setor de energia daquela região cresceu mais do que o dobro dos setores industriais entre 1997 e 2002 e ofertava um salário $80 \%$ maior do que as empresas daquele mesmo setor.

Dessa forma, há fortes indícios de que esse tipo de associação em redes é singular para o desenvolvimento socioeconômico das PMEs, ajudando a minimizar as limitações técnicas e, em especial, financeiras colocadas como empecilhos para uma maior integração de RSC à gestão das empresas (NAHAPIET; GHOSHAL, 1998; SYDOW, 1998; BURT, 2000; MARTELETO; SILVA, 2004).

Diante da polissemia e dificuldade de avaliação, outros aspectos devem ser investigados a fim de mensurar o capital social das empresas. Sydow (1998) descreve alguns pontos importantes que podem servir como balizadores dessa variável nas relações interoganizacionais. São eles: a) frequência e abertura à comunicação interorganizacional; b) multiplicidade das trocas nas relações da rede, como por exemplo, troca de produtos, de informações, de emoções; c) expectativa de continuidade da relação; e d) balanceamento entre autonomia e dependência. Isso significa que em uma relação há uma maior propensão de confiança em redes menores e em redes de empresas com maiores similaridades. Por exemplo, pertença aos mesmos subsistemas sociais.

Ante o exposto, a teoria do capital social parece mais próxima à realidade das PMEs, sobretudo pela facilidade de acesso às vantagens e aos meios para a obtenção delas, o que exige baixo investimento financeiro, por exemplo. 


\section{A Responsabilidade Social como Potencializadora do Capital Social}

O momento de transformação por que o mundo passa exige que as organizações se engajem cada vez mais no desenvolvimento da sociedade na qual fazem negócios. Tal exigência parte do princípio de que as organizações influenciam e são influenciadas pela sociedade em que operam e pelos problemas sociais que as rodeiam (SPENCE; SCHMIDPETER, 2003). Dessa forma, a discussão sobre o papel da empresa como facilitadora de desenvolvimento de uma sociedade ganha cada vez maior importância, sobretudo quando inserida em redes de cooperação com seus diversos stakeholders (CASTELLS, 1999).

No que tange à relação entre capital social e RSC em PMEs, Perrini (2006) aponta que essa ligação pode ser relevante visto a forte influência nas comunidades e a grande flexibilidade e adaptabilidade dessas empresas. Nesse contexto, a primeira pergunta a que se presta é: o capital social é o responsável pelo desenvolvimento de RSC ou essa relação é a inversa? Estudos apontam que não há um consenso entre os pesquisadores sobre essa relação, embora haja uma tendência maior pela segunda premissa (RSC criando capital social) (SANTOS et al., 2006). Nesse caso, verificando essa premissa, uma segunda pergunta ocorre: de que forma maiores práticas de RSC contribuem para o aumento do capital social da empresa e, em última instância, para o reforço da sua capacidade competitiva?

Por meio das ações de RSC, as empresas podem acumular capital social quando, por exemplo, promovem o sucesso na carreira de seus empregados, compartilham informações entre as empresas (PERRINI, 2006). Para Perrini (2006), as PMEs europeias que estão mais envolvidas com RSC estão em redes de bons relacionamentos e, por isso, estão mais habilitadas a retirarem proveitos (não necessariamente econômicos) dessas relações. O autor aponta ainda que, com as ações de RSC, há uma melhora na imagem e na reputação da empresa e a partir disso ocorre um aumento nos níveis de confiança e de crenças compartilhadas entre esses dois atores (empresa e comunidade e/ou clientes).

Moon (2001) advoga nessa mesma direção e afirma que programas de RSC, como por exemplo, os de voluntariado empresarial são importantes geradores de cooperação social, reciprocidade, de aumento de confiança social, de troca de recursos e, portanto, de reforço do capital social. 
No âmbito de uma responsabilidade corporativa mais alargada e de intensificação das relações com os stakeholders, as redes sociais criadas funcionam frequentemente como um recurso importante para consolidar as relações de confiança no contexto da atividade do negócio e, não menos frequente, como uma oportunidade para criar parcerias, aceder a competências complementares ou como fonte de informação relevante para a melhoria do processo de negócio. Spence e Schmidpeter (2003) advogam que o engajamento com a comunidade local, por meio, por exemplo, da doação de dinheiro, de serviço comunitário, ou outras atividades voluntárias constituise como uma forma de manter e desenvolver o capital social das empresas.

As vantagens desses programas, como refere Moon (2001), não são apenas visíveis internamente. A sinergia por elas desencadeadas extravasa o espaço da empresa e das pessoas neles envolvidas, refletindo-se na comunidade de intervenção e nos seus destinatários finais. Nesse domínio é visível o aumento da importância das redes de cooperação, em especial para cimentar as normas sociais e condutas de atuação partilhadas, bem como dar uma sustentabilidade técnica e financeira para esse escope de atividades.

Em contrapartida, outros autores como Fuller e Tian (2006) advogam pela premissa inversa, qual seja: as relações utilizadas para que o capital social desenvolva a responsabilidade social corporativa. Segundo esses autores, ações como ajudar clientes, trabalhar com outros empresários para ajudar a encontrar suas necessidades (nas redes), motivar um equilíbrio entre vida e trabalho dos empregados criam valor para os clientes e atende suas expectativas, ou seja, uma aproximação com os stakeholders facilita a satisfação de suas expectativas, premissa básica de RSC. Putnam (2000) ressalta ainda que as pessoas que geralmente mais confiam em seus parceiros, e, portanto, teriam maior capital social, contribuem mais para unidade e participam mais de ações comunitárias e coletivas. Para Balestro (2002), níveis maiores de interdependência, confiança e colaboração estabelecidos podem ser oportunidades de expansão de contatos horizontais e de negócios para as empresas, o que facilitaria a transmissão e a incorporação de valores socialmente responsáveis.

Diante do exposto, as pesquisas indicam que RSC e o capital social são conceitos que mantêm uma relação e, de certa forma, os complementam. Importa com isso compreender quais são os ganhos da aproximação das PMEs para seus stakeholders, em especial, para a comunidade, os fornecedores e os empregados que são fundamentais para a sobrevivência e a manutenção 
no mercado de qualquer empresa. Além disso, o fato de ter baixos custos, já que em um primeiro momento não se exige uma formalização técnica (mensuração, instrumentos específicos, consultoria, etc.), nem mesmo grandes investimentos financeiros, favorece e muito o desenvolvimento de relacionamentos de PMEs em redes sociais e a partir disso uma participação maior em ações socialmente responsáveis.

\section{Considerações Finais}

As empresas não agem num vácuo, nem mesmo podem agir sozinhas no mercado. Necessitam de clientes, parceiros, fornecedores, empregados e outros atores que permitam seu progresso financeiro. Assim, a proposta deste texto foi mostrar como a gestão socialmente responsável e a teoria do capital social possibilitam essa aproximação e ainda os benefícios da aplicação desses conceitos para as pequenas e médias empresas.

Esses conceitos não representam uma solução mágica e pronta para o desenvolvimento das PMEs e nem a integração de práticas de RSC à gestão dessas empresas seja elementar e de rápida implementação. Muito há de se caminhar e encorajar esse empresariado de que existem evidências de sucesso e ainda certas garantias de que a integração em redes de empresas e a integração de RSC à gestão empresarial é possível. Verschoore (2004) afirma que há várias barreiras a superar como a mentalidade individualista dos empresários, a heterogeneidade de expectativas dos participantes das redes e, em especial, o baixo acúmulo de capital social, sobretudo o que se refere ao compartilhamento de normas e valores entre os empresários. Para o autor, esses obstáculos distanciam os agentes participantes, dificultam o empreendimento de ações conjuntas e entravam a disseminação de ideias de cooperação em toda a sociedade.

Desse modo, pesquisas futuras devem buscar a compreensão de como o capital social pode ser desenvolvido nas PMEs, mais especificamente, como os níveis de confiança entre empresa/empresa, empresa/empregado e empresa/sociedade podem ser potencializados, reativados (parte-se da premissa de que toda sociedade já tem seu capital social) e a partir disso incorporados à gestão socialmente responsável dessas empresas. Para tal, a investigação das variáveis indicadas por Sydow (1998) podem significar bons indicadores: posições e papéis dos atores no relacionamento com a empresa, 
frequência e abertura à comunicação entre os parceiros da empresa e densidade das ligações da empresa a outras empresas, clientes, comunidades e fornecedores. Ademais, aspectos intraorganizacionais, como a existência de estruturas flexíveis, pouco hierárquicas, e com abertura perante a mudança podem ser elementos facilitadores para a integração do conhecimento dos stakeholders nas PMEs.

Isso feito, as pesquisas que envolvam redes, PMEs e RSC podem contribuir para dois aspectos. O primeiro se refere ao fato de que diante da escassez, apontada na literatura, poderão ser desenvolvidos instrumentos específicos de e para avaliação das redes de pequenas e médias empresas e seu potencial para a integração de RSC à sua gestão. Esses instrumentos poderão ser bem mais simples e adaptáveis do que os que são propostos como decorrência da teoria dos stakeholders, o que poderá contribuir ainda para um avanço no caráter essencialmente filantrópico e assistencialista hoje observado entre as PMEs. Outra contribuição aponta para o desenvolvimento de conhecimento que subsidie parcerias mais eficientes e efetivas entre as empresas (COLEMAN, 1988; CASTELLS, 1999; PERRINI, 2006). Acresce que estudos (ZADEK, 2001; AYUSO et al., 2006) têm demonstrado que o estabelecimento de um diálogo interativo e a integração do conhecimento dos stakeholders em uma ótica da responsabilidade social que incorpore os desafios ambientais e sociais às necessidades da comunidade, potencia o processo de inovação através da integração de novas ideias na gestão do negócio, reforçando a capacidade competitiva das empresas. Além disso, a compreensão dos efeitos das redes de cooperação pode favorecer o desenvolvimento regional, a formulação de políticas públicas para o desenvolvimento de micro, pequenas e médias empresas e ainda para o fortalecimento da gestão de RSC em PMEs e, sobretudo, contribuir para o desenvolvimento de agrupamentos de responsabilidade corporativa, com benefícios competitivos ao nível macro, ultrapassando de uma visão restritiva da responsabilidade social centrada no "business case". Há ainda muito que se caminhar para tornar a ideia de RSC na PMEs uma realidade, sobretudo quando em redes de cooperação, estruturalmente organizadas em agrupamentos de responsabilidade corporativa. 


\section{The Importance of Corporate Social Responsibility for Advertising of Social Capital in Small and Medium Enterprises}

\section{Abstract}

Social, economic and technological changes within the modern world have transformed the role of companies before society and, through this prism, the issue of Corporative Social Responsibility stands out. However, for small and mediumsized companies, there are several limitations which prevent their management to benefit from a bigger integration in such matter. As a means of overcoming obstacles, a broader application of social capital concept is been attemptively set through stronger trust bonds, belief and norm sharing, and social network interaction. Therefore, the present study aims to present the theory of social capital and link it to Corporative Social Responsibility and comprehend their interactions, applicability and gains applied to small and medium enterprises. It is also pointed out some variables which may serve to measure Corporative Social Responsibility and Social Capital in future researches.

Key words: Social responsibility. Social capital. Small and medium enterprises.

\section{Referências}

ADLER, Paul; KWON, Seok-Woo. Social capital: the good, the bad, and the ugly. In: Lesser, Eric L. Knowledge and social capital: foundations and applications. Woburn: Butterworth-Heinemann, 2000, p. 89-115.

ARAÚJO, Maria. Capital social. Rio de Janeiro: Jorge Zahar, 2003.

AYUSO, Silvia; RODRÍGUEZ, Miguel; RICART, Joan. Responsible competitiveness at the "micro" level of the firm. Using stakeholder dialogue as a source for new ideas: a dynamic capability underlying sustainable innovation,

Corporate Governance, v. 6, n. 4, p. 475-490, 2006.

BALESTRIN, Alsones; VARGAS, Lilia. A dimensão estratégica das redes horizontais de PMEs: teorizações e evidências. RAC, edição especial, p. 203227, 2004. 
BALESTRO, Moisés. Confiança em rede: a experiência da Rede de Estofadores do Pólo Moveleiro. Dissertação de Mestrado. Universidade Federal do Rio Grande do Sul, Porto Alegre: Universidade Federal do Rio Grande do Sul, 2002.

BOURDIEU, Pierre. The forms of capital. In: Richardson, J. G. Handbook of theory and research for the sociology of education. New York: Greenwood, 1986, p. 241-258.

BURT, Ronald. The network structure of social capital. In: Sutton, R. I.; Staw, B. M. Research in Organizational Behavior. Greenwich: JAI Press, 2000, p. 345-423.

CASTELLS, Manuel. A sociedade em rede. São Paulo: Paz e Terra, 1999.

COLEMAN, James. Social Capital in the creation of human capital. American Journal of Sociology, v. 95, p. 95-120, 1988.

FUKUYAMA, Francis. Trust: the social virtues and the creation of prosperity. New York: Free Press. 1995.

FULLER, Ted.; TIAN, Yumiao. Social and symbolic Capital and responsible entrepreneurship: an empirical investigation of SME narratives. Journal of Business Ethics, v. 67, p. 287-304, 2006.

GRAAFLAND, Johan; VAN DE VEN, Bert; STOFFELE, Nelleke. Strategies and instruments for organising CSR by small and large business in the Netherlands. Journal of Business Ethics, v. 47, n. 1, p. 45-60, 2003.

GRANOVETTER, Mark. The strength of weak ties. American Journal of Sociology, v. 78, n. 6, p. 1360-1380, 1973.

HABISCH, André. Social responsibility, social capital and SMEs. In: Spence, Laura.; Habisch, André.; Schmidpeter, R. Responsibility and social capital: the world of small and medium sized enterprises. Hampshire: Palgrave Macmillan, 2004, p. 25-34.

JENKINS, Helled. Small business champions for corporate social responsability. Journal of Business Ethics, v. 67, p. 241-256, 2006.

MARTELETO, Regina; SILVA, Antônio. Redes e capital social: o enfoque da informação para o desenvolvimento local. Ciência e informação Brasília, v. 33, n. 3, p. 41-49, 2004. 
MELO NETO, Francisco; FROES, César. Gestão da responsabilidade Social Corporativa: o caso brasileiro. Da filantropia tradicional à filantropia de alto rendimento ao empreendedorismo social. Rio de Janeiro: Qualitymark, 2001.

MONTANA, Jennifer; NENIDE, Boris. The evolution of Regional Industry clusters and their implications for sustainable economic development. Two cases illustrations. Economic Development Quaterly, v. 22, n.4, p. 290-302, 2008.

MOON, Jeremy. Business Social Responsibility: a source of social capital? Reason in Practice, v. 1, n. 3, p. 35-45, 2001.

NAHAPIET, Janine; GHOSHAL, Sumantra. Social capital, intellectual capital and the organizational advantage. The academy of management review, v. 23, n. 2, p. 242-266, 1998.

PELIANO, Ana M. A iniciativa privada e o espírito público. um retrato da ação social das empresas no Brasil. Instituto de Pesquisas Econômicas Aplicada, Brasília, 2002.

PERRINI, Francesco. SMEs and CSR theory: evidence and implications from an italian perspective. Journal of Business Ethics, v. 67, p. 305-316, 2006.

PUTNAM, Robert. Bowling alone: the collapse and revival of american community. Simon and Shuster: New York, 2000.

REGO, Arménio. Gestão Ética e Responsabilidade social das empresas. Principia: Cascais. Portugal, 2003.

RING, Peter S.; VAN DE VEN, Andrew. Developmental processes of cooperative interorganizational relationatioships. Academy of management review, $v$. 19, n. 1, 90-118, 1994.

SAKO, Mari. Does trust improve business performance? In: Lane, Christel e Bachmann, Reinhard. Trust within and between organizations.

Conceptual issues and empirical applications. p. 88-117. Oxford: Oxford Universtity Press. 1998.

SANTOS, Maria João; SANTOS, Ana M.; PEREIRA, Elisabete N.; ALMEIDA, José L. Responsabilidade Social nas PME. Lisboa: RH, 2006. 
SERVIÇO BRASILEIRO DE APOIO ÀS MICRO E PEQUENAS EMPRESAS SEBRAE. Boletim estatístico de micro e pequenas empresas, 2005.

SOUTHWELL, Clare. Engaging SMEs in community and social issues. In: SPENCE L. J.; Habisch, A.; Schmidpeter, R. Responsibility and social capital: the world of small and medium sized enterprises. Hampshire: Palgrave Macmillan, 2004, p. 96-111.

SYDOW, Jorg. Understanding the constitution of interorganizational trust. In: LANE, C.; BACHMAN, R. Trust within and between organizations. New York: Oxford University Press, 1998, p. 31-63.

SPENCE, Laura. J.; SCHMIDPETER, René. SMEs, social capital and commom good. Journal of Business Ethics, v. 45, p. 93-108, 2003.

TEIXEIRA, Rivanda M. Small business and social responsibility in Brazil. Cranfield. 341 f. Tese de doutorado da Cranfield University. Cranfield: Cranfield University, 1996.

UNITED NATIONS INDUSTRIAL DEVELOPMENT ORGANIZATION (UNIDO). Corporate Social Responsibility: Implications for Small and Medium Enterprises in Developing Countries. Vienna: UNIDO, 2002.

VERSCHOORE, Jorge R. Redes de cooperação: uma nova organização de pequenas e médias empresas no Rio Grande do Sul. Porto Alegre: FEE, 2004.

VISSER, Wayne. Corporate Social Responsibility in developing countries. In: GRANE, A.; MCWILLIAMS, D.; MATTEN, J.; MOON, D.; SIEGEL. The oxford handbook of corporate social responsibility. Oxford: Oxford University Press, 2008, p. 473-499.

WERNER, Andrea.; SPENCE, Laura. J. Literature review: social capital and SMEs. In: SPENCE, L. J.; HABISCH, A.; SCHMIDPETER, R. Responsibility and social capital: the world of small and medium sized enterprises. Hampshire: Palgrave Macmillan, 2004, p. 7-24.

WORTHINGTON, Ian; RAM, Monder; JONES, Trevor. Exploring corporate social responsibility in the UK asian small business. Journal of Business Ethics, v. 67, p. 201-217, 2006.

ZADEK, Simon. The civil corporation. The economy of corporate citizenship. London: Erthscan Publications, 2001. 